\title{
FTIR and ESEM Analysis of soil moisture Microscopic conservation feature with liquid membrane
}

\author{
Jian $\mathrm{Gu}^{1,2}$, Liang Hao ${ }^{2,3}$, Pengfei Huang ${ }^{1,2}, \mathrm{Na} \mathrm{Tong}^{4}, \mathrm{Xue} \mathrm{Li}^{1,2}$, Guanghua Yin ${ }^{1, a}$ \\ ${ }^{1}$ Institute of Applied Ecology, Chinese Academy of Sciences, Shenyang, 110016, China \\ ${ }^{2}$ University of Chinese Academy of Science, Beijing, 100039,China \\ ${ }^{3}$ Institute of Policy and Management, Chinese Academy of Sciences, Beijing, 100190,China \\ ${ }^{4}$ Shenyang Institute of Engineering, Shenyang, 110136, China.
}

\begin{abstract}
Liquid membrane applied in soil has a good soil moisture conservation and evaporation suppression performance. Application of spectrum analysis technology to understand its structure and micro morphological characteristics will be help to reveal the soil moisture conservation mechanism of liquid membrane. In this paper, we used the three types of liquid membrane as the research object based on the laboratory preparation, with infrared spectrometer (FTIR) and environmental scanning electron microscope (ESEM) as the means, analysis the soil moisture mechanism of liquid membrane.The results showed that the -OH between the CMC and PVA generated intermolecular hydrogen bonds, the formation of hydrogen bonds between molecules of the two components strengthened the two-phase's compatibility, increasing the liquid membrane's effective groups and forming a dense mesh structure.ESEM observation showed that liquid membrane can effectively cementing soil particles, generating the soil-membrane structure, reducing soil moisture to evaporate, improve soil moisture conservation performance. keywords: liquid membrane; microscopic mechanism; FTIR;ESEM
\end{abstract}

\section{Introduction}

Water shortage is the key factor to restrict the agricultural sustainable development in arid and semiarid regions, plastic mulching technique is an effective way to solve the problem [1]. Since 1979, our country imported polyethylene mulch from Japan, its rapid development has made significant yield effect. However, polyethylene-mulch is a kind of macromolecular hydrocarbon, which is insoluble in water and not readily degrade in the soil, it has brought serious white pollution to the environment because of wide and long-term using in the agricultural production ${ }^{[2]}$. Therefore, developing a environmentally friendly material to replace polyethylene mulch has become an urgent need for the development of ecological agriculture. Liquid membrane is a new type of covering products with organic polymer as the main material, which is the recent development and application of agricultural production ${ }^{[3]}$. The product sprayed on the surface can effectively cementing soil particles, generating the soil-membrane structure, reducing soil moisture to evaporate, improve soil moisture conservation performance ${ }^{[4]}$. Research shows that ${ }^{[5-}$ ${ }^{7]}$,Liquid membrane is showing superiority in the economy (reduce the amount), environment (security clearances dose), soil conservation (improved soil structure), irrigation efficiency (increase infiltration / reduce runoff), etc. However, the current researches on soil moisture conservation performance for liquid membrane mainly adopt the method of field experiment fixed-point observation, don't sets forth the mechanism of soil moisture conservation from the micro structure of liquid membrane ${ }^{[10-13]}$, the research is not deep enough. The successful application of modern test technology Such as X-ray diffraction, infrared spectrum, differential thermal analysis, electron microscope, nuclear magnetic resonance (NMR) and electron spin resonance, etc, in the analysis of soil related components and structural analysis provides a way for the research on the microstructure of liquid membrane.

In view of this, this article try to infrared spectrometer (FT-IR) and environmental scanning electron microscope (ESEM) as the means, analysis the soil moisture mechanism of liquid membrane, try to reveal the soil moisture conservaton mechanism of liquid membrane from spectroscopy, so as to provide reference for the similar research and using for reference.

\section{Experimental part}

\subsection{Preparation of liquid membrane}

Liquid membrane is based on polyvinyl alcohol (PVA) and sodium carboxymethyl cellulose (CMC) as the main raw materials, crosslinking agents, defoamers, glycerin and water as the secondary raw materials, three types of liquid membrane were prepared and labeled as 1 (PVA : $\quad \mathrm{CMC}=3: 1$ ), 2 (PVA : $\mathrm{CMC}=1: 1$ ) and 3 (PVA : $\mathrm{CMC}=3: 1$ ). Preparation methods are as follows:

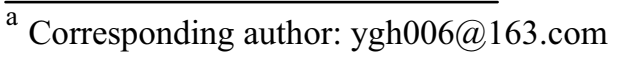


The pure PVA powder and pure CMC powder were mixed in deionized water according to three ratios, bathing for $3 \mathrm{~h}$ at $80{ }^{\circ} \mathrm{C}$ in the autoclave water, at the same time, constant stirring, the speed is $100 \mathrm{r} / \mathrm{min}$, the quality of the solution is obtained by $8 \%$. After completely dissolved, add other auxiliary materials, $0.5 \mathrm{~h}$ reaction, and the liquid membrane is prepared after cooling.

\subsection{Test methods}

\subsubsection{Structure determination}

The liquid membrane and PVA and CMC liquid flows into the watch glass, film casting, placed on the bench at room temperature for three days, and then alternate. Use the Nicolet 6700 Fourier infrared spectrometer (FTIR) produced by the Thermo Fisher company for functional characterization of liquid membrane, measuring the absorption spectrum of liquid membrane and structural analysis.

\subsection{2 soil-liquid membrane microscopic moisture morphometric}

The three kinds of liquid membrane were dissolved in water at a concentration of 1:20, using $300 \mathrm{~kg} / \mathrm{hm} 2 \mathrm{of}$ liquid.After thorough stirring, the solution was sprayed on the surface of the soil. An identical amount of water was sprayed as a control. The soil was sampledtwice a month for four monthsafter spraying.A QuantaTM 250 ESEM produced by American FEI Company was used to determine and analyze the micromorphology of the liquid membrane-treated soil.

\section{Results}

\subsection{Structure Determination}

From the results of Figure 1, it can be seen that the PVA chain is flexible, while the CMC molecular chain contains a large number of ring structures.Although there is a big difference between the two groups of macromolecular chain structure, both of the PVA and the CMC molecules have a hydroxyl group, intermolecular hydrogen bonds between them can improve the compatibility between PVA and CMC.

In pure PVA film, $3251 \mathrm{~cm}^{-1}$ is stretching vibration peak of $-\mathrm{OH}, 2906 \mathrm{~cm}^{-1}$ is $-\mathrm{CH}$ stretching vibration of, $1085 \mathrm{~cm}^{-1}$ is symmetrical and asymmetrical C-O absorption peak; Stretching vibration of pure CMC film $\mathrm{OH}$ appears in $3356 \mathrm{~cm}^{-1}, 2941 \mathrm{~cm}^{-1}$ is $-\mathrm{CH}$ stretching vibration peak, $1600 \mathrm{~cm}^{-1}$ is $-\mathrm{COONa}$ groups $\mathrm{C}=\mathrm{O}$ symmetric and asymmetric vibration absorption peaks. When the CMC and PVA blended -OH on PVA molecular chains will bind with ionized carboxylate, compared to $\mathrm{Na}^{+}$, - $\mathrm{OH}$ has the functions of providing electronic, so $\mathrm{C}=\mathrm{O}$ infrared absorption peak moves to the high number of wave.For liquid film, the position of the $\mathrm{OH}$ peak was changed, and it appeared in the $3303 \mathrm{~cm}^{-1}, 3265 \mathrm{~cm}^{-1}$ and $3200 \mathrm{~cm}^{-1}$ also shows CMC -OH and polyvinyl alcohol (PVA) -OH also produce intermolecular hydrogen bonds, the formation of intermolecular hydrogen bonds between the two components of molecular strengthen the compatibility between two phase, so that the liquid membrane formed a dense mesh structure, favorable for water and soil moisture conservation.

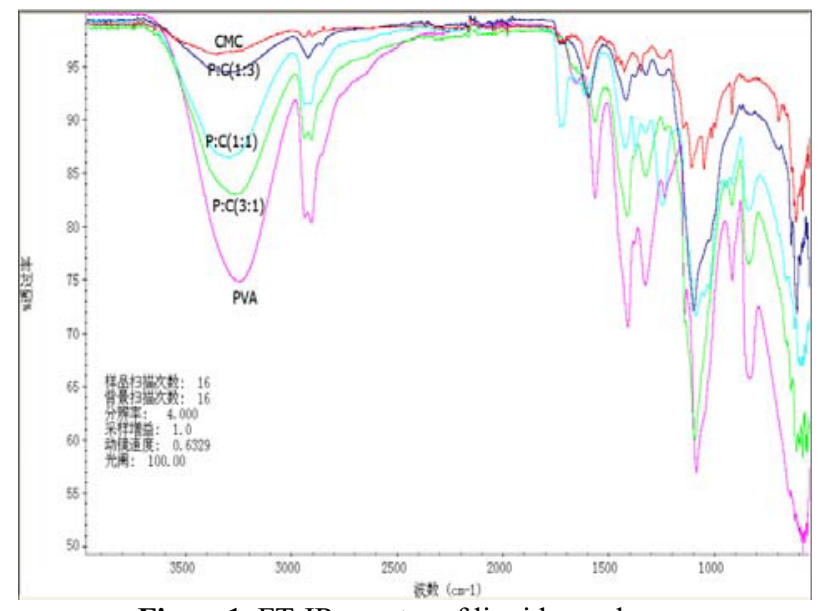

Figure1. FT-IR spactra of liquid membrane

\subsection{Soil membrane structure Micromorphological}
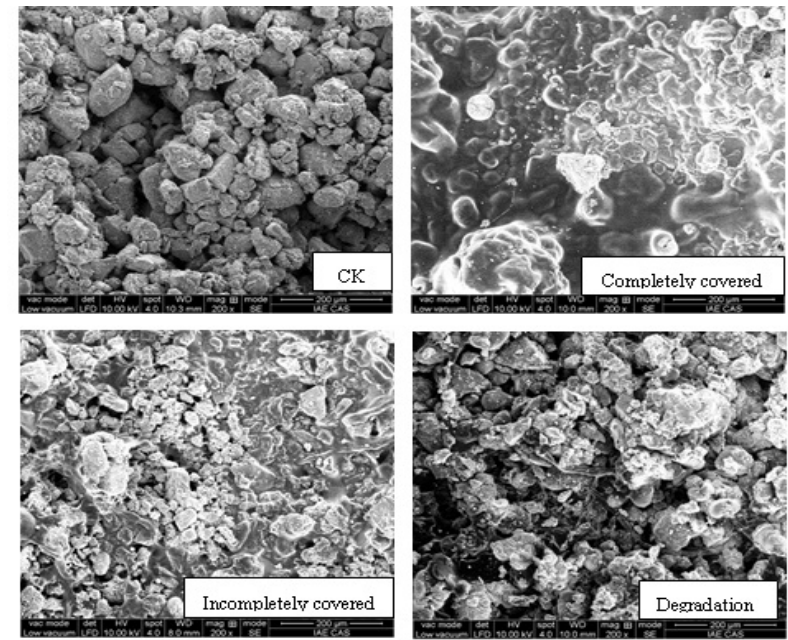

Figure 2.The soil surface micromorphology with different treatments

Figure 2 shows that the control group and the soil surface covered with liquid membrane have completely different structural characteristics.

The figure in upper left corner is the control, the soil surface layer of soil particle accumulation is compact, small pore, see more small particles in the larger pore filling, the formation of soil crust. The surface of the soil covering with liquid membrane is different,pictured top right completely covered is an ideal way to cover,liquid membrane completely wrapped in the soil particle surface, and can be connected to each other to form a closed membrane structure of soil;Pictured left partially covered, the soil surface is not completely covered, large pores, poor soil moisture results; the lower right picture shows 
the degradation effect after four months,most of the liquid membrane covering the soil surface degrade, only remains connection between part soil particles, Basically lost soil moisture conservation performance.

\subsection{Three types of liquid membrane covering effect}

Figure 3 shows the effect of the liquid membrane covering for half of a moon, Wherein the upper left corner picture shows the effect of the 2 nd liquid membrane, film is completely wrapped in the soil surface, good sealing effect;top right photo shows the 2nd liquid membrane coverage effect, which is not complete, there are more pores, poor sealing effect; The lower left corner figure shows the effect of the 2 nd liquid membrane,the material in the picture is less, more common filamentous material, good connection effect, but the effect is not ideal.The main cause of this phenomenon is that the different components of the liquid membrane.The viscosity of sodium carboxymethyl cellulose solution is relatively high, and the mobility is poor;while the polyvinyl alcohol solution flow better ductility.Therefore, the cover effect of 1 nd liquid membrane which containing most polytene is best,no. 2 and no. 3, liquid membrane covering effect is poorer.
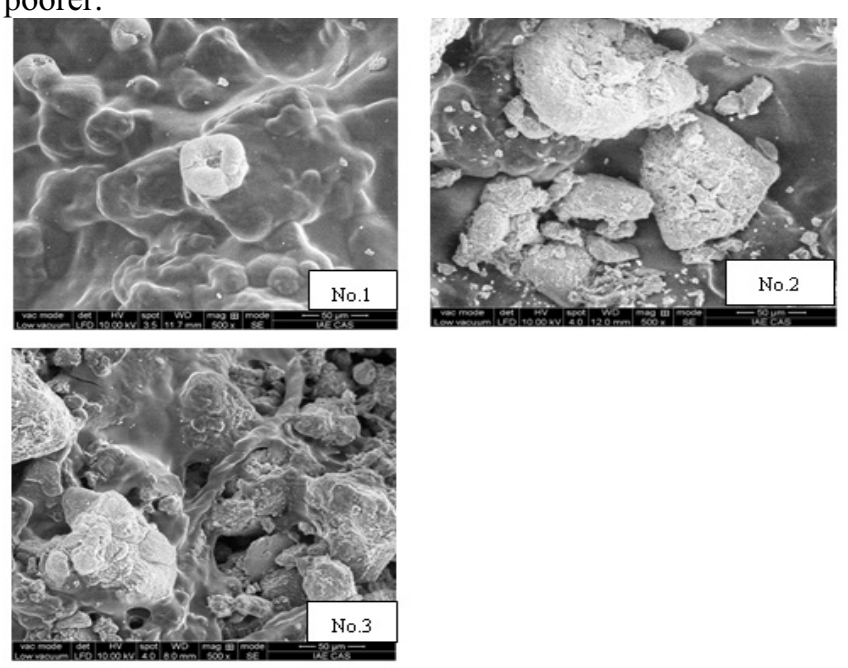

Figure 3. Three types of liquid membrane coverage of the soil surface micromorphology

\subsection{The connection of the liquid membrane with soil particles}

Figure4 shows the combination method of liquid membrane and soil particles. The hydrophilic group of the liquid membrane can be formed by hydrogen bonding with many substances, which can produce a bond between the two particles, a molecule can bond a few particles at the same time usually.As shown in the figure,under the effect of flocculant sticky, large particles surface adsorb a lot of fine particles in the soil. There are a lot of connection between the floc particles,forming a lattice-like structure.In the soil, the pore is large and the connectivity is good. The sticky substance formed after dissolution similar to the hydrogen bonding can connect two or more soil particles to form a stable structure. The "key" that plays a role of sticky flocculant can be found everywhere in the soil surface, which has a strong adhesive force. Its role as the the chemical bond in molecular structure, can make the soil particles contact no longer point to point between each other, but the connecting way of lattice frame, make the soil structure more stable and lattice frame of pore in soil not easy to damage. Liquid membrane can cover soil particles,their component filaments can bond soil particles.The improvement of soil physical and chemical properties and the accumulation of organic matter promote the formation of soil structure and enhanced the surface stability.

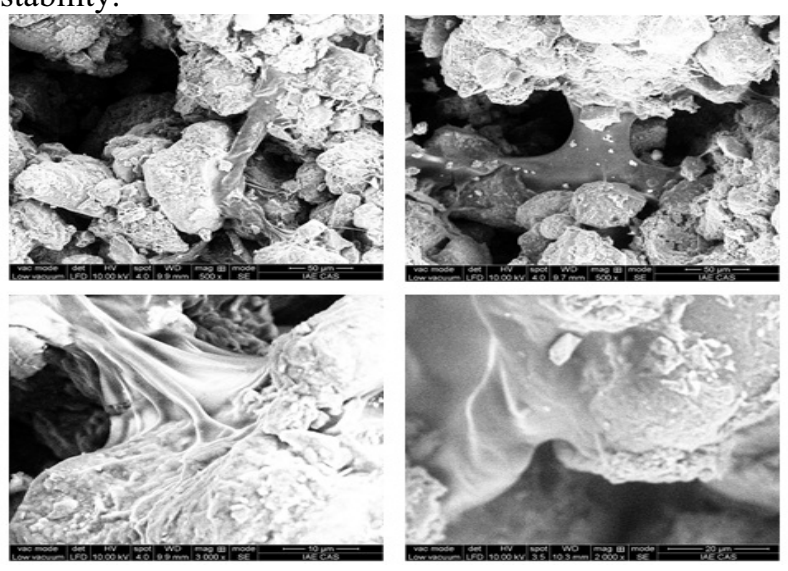

Figure 4. the connection of the liquid membrane with soil particles

\section{4 conclusion}

(1) The $-\mathrm{OH}$ in $\mathrm{CMC}$ which is the main component of the liquid membrane and the $-\mathrm{OH}$ in PVA can produce intermolecular hydrogen bonds, the formation of hydrogen bonds between the two components of molecular enhanced the compatibility between the two phase, and reached the purpose of increasing the effective group in liquid membrane and the formation of dense network.

(2) Liquid membrane can effectively bond soil particles, forming a structure of the soil and liquid membrane and reduce evaporation, increase soil moisture conservation performance.In comparison with the micro morphology of different time, it was found that lliquid membrane degradation happens, will not cause pollution of soil environment.

(3)FTIR and ESEM can effectively reveal the soil moisture conservation mechanism of liquid membrane. Through the observation of the soil and liquid membrane in different periods, the degradation of liquid film can be inferred, which can provide theoretical basis for the popularization and application of liquid membrane. 


\section{Acknowledgements}

The authors would like to express their sincere thanks to the editor and anonymous reviewer's comments and suggestions for the improvement of this paper.

This research was supported by the Special Fund for Agro-scientific Research in the Public Interest (201303125), the National Major Technology Program (2013BAD05B07; 2011BAD16B12; 2012BAD04B03) and the science and technology research projects of Liaoning province (2015103018).

\section{References}

1. Wang X., Xu G.B., Ren Z.G., Zhang Z.J., Jian Y.F., Zhang Y.M.Chinese J. Eco-Agr., 15(1),78-81(2007).

2. Yan C.R., Mei X.R., He W.Q., Zheng S.H. T. CSAE, 22( 11), 269-272(2006).

3. Vlad S, Vlad A, Oprea S. Eur. Polym. J. 38(4), 829-835(2002).

4. Cascaval C, Rosu D, Rosu L. Polym. Test. 22(1), 45-49(2003).

5. Zhang J., Yao Y.Q., Lu J.J., Wang Y.H., Li J.H., Wang C.H. Chinese J. Soil Sci. 36(4),638-640(2005).

6. Yin G.H., Tong N., Hao L., Gu J., Liu Z.X. J. Soil Water Conserv. 26(4), 204-207(2012).

7. Li C.X.,Zhou X.G., Qiang X.M., Guo D.D., Liu Z.G., Zhang J.P. Maize Sci., 18(3), 108-112 (2010).

8. Lentz, R.D. and R.E. Sojka. Soil Sci. 158, 274 282(1994).

9. G.F.Fanta,E.B. Encyclopaedia Polym. Sci. Techno.,2(1), 665 (Wiley-Interscience,NY,1977).

10. Yin, G., L. Hao, Y. Zhao, J. Gu and Z. Liu. Chinese J. Eco. 32(6), 1405-1411 (2013).

11. Wang C.H.,Zhang J.,Li J.h.,Ding Z.Q.,Wang Y.H.,Yao Y.Q.,Lu J.J. Anhui Agric. Sci. 34(14), 3289-3290(2006).

12. Li, Y., Z. Wang, J. Zhang, X. Zheng, W. Li and G. Jiang. Aca. Agric. Boreali-Occidentalis Sin. 23(12), 110-115(2014).

13. Zhang, H., J. Li, Z. Jia, T. Zhang, X. Hou and P. Zhang. Agricul. Res. Arid Areas 30(2), 93100(2012). 